Bogotá

\title{
VIGENCIA DEL DIPTONGO HISPÁNICO huij
}

\section{INTRODUCCIÓN}

Reflexiones a través de la cátedra de fonética en pregrado y postgrado motivaron la gestación de esta investigación sobre la presencia en lengua hispánica actual de un diptongo más $\left(15^{\circ}\right)$, presente en la combinación $u i$, en palabras tal vez pocas pero suficientes que muestran una configuración fónica muy específica en la concurrencia vocálica referida.

Las fonéticas españolas tradicionales habían venido consagrando 14 diptongos y daban por inexistente el diptongo [ui]], realización vigente y común en el mundo hispánico, casi que confirmada previamente a través del análisis impresionístico (auditivo) en vocablos como muy, huy, Rny, Cocuy, cuy, Jujuy, etc. Pero para dilucidar el hecho nos propusimos llevar la demostración de la misma evidencia al examen acústico experimental con un material lingüístico seleccionado y 20 informantes de diferentes edades, diferentes sexos y diferente formación académica, pues de entrada nos parecía con certeza que la concurrencia ui no siempre es de ejecución fónica [wi] y era pues necesario despejar esta inquietud científica.

Examinadas 45 muestras y realizados los conteos estadísticos, se evidenció que en un $88 \%$, el diptongo [ $u$ i $]$ es una realidad, hasta hoy desestimada, pero con toda una evidencia en su favor que lo hace mucho más caracterizado que [ou ], que es la menos representativa de nuestras concurrencias.

Recurrimos no a la observación impresionística (mayor ennegrecimiento) del segmento de la concurrencia /ui/ sino a la medición y comparación de cúspides pertenecientes a $[u]$ y a $[i]$ con la satisfactoria evidencia de que en el material examinado predomina en un altísimo porcentaje el diptongo [ui] y no [wi], situación que se identifica con el criterio auditivo de todos los informantes utilizados, quienes luego de sus grabaciones personales, espontáneas y desprevenidas, fueron del parecer que el acento prosódico (intensivo en este caso) recaía sobre la $u$ y no sobre la $i$.

En el comienzo del trabajo nos pareció necesario hacer el recuento del vocalismo español y mencionar las concurrencias para entrar con más piso a la discusión central.

Constituyen un buen aporte la inclusión o presentación del material lexicográfico hispanoamericano de términos con $\bar{u} y$, así como los 5 cuadros de segmentos Amplitude display cuyo sector izquierdo (elevación) es el decibelaje correspondiente a $[u]$ y su continuidad con sus respectivas elevaciones, a [i]; la extracción de los segmentos intensivos se realizó por el método de calcado de los sonogramas. Cinco de los 45 
sonogramas se han mostrado también como ratificación del fenómeno que se pretendía probar.

En la convicción de que a partir de esta investigación se plantea con propiedad y evidencia la existencia de un $15^{\circ}$ diptongo, este trabajo queda indicado para los profesores de lengua española en escuelas medias; igualmente puede aprovechar a los profesores y estudiantes de las ciencias fonéticas de nivel universitario, así como también a autores y editorialistas quienes pueden, sin duda alguna, incluir en las nuevas clasificaciones vocálicas hispánicas el diptongo [ui] como realización plenamente válida en el español moderno.

Finalmente, la realización de este trabajo sólo ha sido posible mediante la existencia y utilización del Laboratorio de Fonética Experimental del Instituto Caro y Cuervo y la permanente ayuda científica que desde 1975, como becario, vengo recibiendo de la Alexander von Humboldt Stiftung de la República Federal de Alemania; sin su contribución y directrices me hubiera sido imposible emprender en estos modestos trabajos de investigación, que son continuidad y resultado de los planes trazados por el IKP de Bonn en 1975/76.

\section{DESCRIPCIÓN SINCRÓNICA DE LA DIPTONGACIÓN ESPAÑOLA}

Anota don Tomás ${ }^{1}$ que los diptongos decrecientes están formados por la

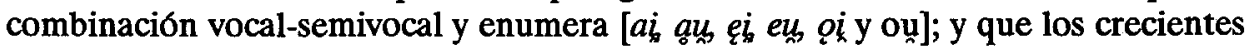
están dados por las combinaciones de semiconsonante más vocal, así: [ja, je, jo, ju, wa, we, wo y wi]. En suma, para Tomás Navarro sólo existen 14 diptongos hispánicos.

Gili Gaya ${ }^{2}$ menciona como diptongos crecientes [ja, je, jo, wa, we, wo] y como

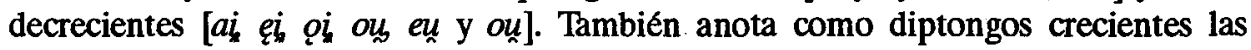
combinaciones de las dos vocales débiles (entiéndase modernamente como cerradas) $i$, $u$ en donde según él, el primer elemento es semiconsonántico y el segundo, es el núcleo silábico, es decir, $[j u$, wi $]$. Más adelante anota que estos dos últimos fonos ofrecen variedad de situaciones dialectales que hacen del primer elemento el núcleo silábico para lo cual ejemplifica, así: tri-un-fo, ju-i-cio, agregando, además, que, en general, en España, la pronunciación de muy es [ $\mathrm{mwi}]$, la cual dialectalmente en la Península puede llegar a ser [mul]. Con esto último, referido a la posibilidad diptongal de $i, u$, no estamos de acuerdo.

Quilis ${ }^{3}$ se refiere, al igual que Tomás Navarro, a 8 diptongos crecientes [ja, je, jo, $w a, w e, w o, j u, w i]$ e incluye como ejemplo de [wi] el término muy; es decir, se acoge

1 Tomás Navarro, Manual de pronunciación, p. 65.

2 Samuel Gili Gaya, Elementos de fonética general, p. 114.

3 Antonio Quilis, Curso de fonética y fonología, pp. 65-66. 
a la posición de Gili Gaya. Igualmente, incluye o sostiene la existencia de 6 diptongos

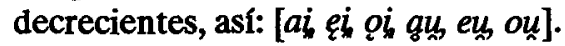

A través de la revisión de estos tres tratadistas hemos encontrado situciones fónicas de marco peninsular que distan y difieren de lo general y común en el marco hispanoamericano en cuanto a pronunciación. Veamos el estimativo que ellos hacen de hiato o diptongo, sobre todo, en las siguientes concurrencias vocálicas, de las cuales presentamos sus correspondientes particiones silábicas: [bi-a-xe], [su-a-be] y [kru-el], (Navarro, p. 67), de las cuales dice, se pueden convertir en las siguientes sinéresis: [bja-xe], [swa-be] y [krwel]. Rehu-sar, parte silábicamente Quilis (p. 68), lo que nosotros oímos como re-nu-sar. Inversamente, Quilis considera como hiatos (p. 71) las palabras siguientes, las cuales se pronuncian en América como diptongo corriente. Ejemplos: di-a-rio, vi-á-ti-co, pi-a-no, bi-e-la, bi-ó-xi-do, cu-o-ta, re-u-ma. En todo caso, oídas algunas personas de procedencia española residentes en Colombia se pudo observar que prefieren la pronunciación hispanoamericana y no la pronunciación propuesta por Navarro y Quilis, presentada arriba con sus respectivas particiones silábicas.

Examinada así, brevemente, la teoría tradicional del diptongo hispánico, pasemos ahora a un breve capítulo sobre la presencia de una combinación vocálica muy frecuente por estas latitudes hispanoamericanas: La concurrencia uy en palabras, como: muy, Ruy, Cocuy, huy, cuy, juy, entre otras tantas.

\section{RECUENTO DE LOS DIPTONGOS HISPÁNICOS ACTUALES}

\section{DIPTONGOS CRECIENTES}

(Semicons. C/2 + VP o silábica)
1. [ja] Viajero
2. [je] Vienesa
3. [jo] Fiordo
4. $[j u]$ viuda
5. [wa] cuadro
6. [we] fuente
7. [wo] acuoso
8. [wi] intuición 
DIPTONGOS DECRECIENTES

VP o silábica + V/2 (semivocal)

9. [aị] baile

10. [Ei] reina

11. [ख]] Moisés

12. [au ] Aurora

13. [eu ] eutanasia

14. $[o u]$ Bou

15. [ui] Por demostrar

\section{PRESENCIA DE UN DÉCIMO QUINTO DIPTONGO HISPÁNICO (MUESTRAS LÉXICAS DE AMÉRICA)}

A lo largo de una lenta observación no solamente al nivel del habla callejera, familiar y académica, sino también en juiciosas observaciones de la clasificación estructural que se había venido presentando sobre el fenómeno vocálico ui, pudimos notar con sorpresa dos aspectos muy importantes al respecto.

A. Que los hablantes hispanoamericanos, en ejemplos, como: Muy, Ruy, Cocuy, cuy, huy, etc., según nuestro examen impresionístico, ejecutan el mayor acento intensivo sobre la vocal $u$ y no sobre la $i$, determinando con ello, la constitución de esa vocal como el centro o elemento núcleo-silábico, y de la $i$ como elemento periférico o marginal, en este caso con carácter semivocálico. Posteriores análisis al respecto sobre el mayor carácter intensivo de la vocal $u$, nos dieron confirmadamente la razón de que en Hispanoamérica, e inclusive en la misma España, se presenta con gran vigencia la existencia del diptongo [ui].

B. Examinados los presupuestos teóricos existentes en los textos de fonética española, encontramos, primero: que combinaciones, como [ou], tan pobremente representadas en la lengua, sólo existe una palabra que lo contiene (bou), son un diptongo efectivamente confirmado y, segundo, que existiendo una serie de términos bastante surtida con diptongo [ $u i]$ como las ya mencionadas, este diptongo decreciente no está propuesto normativamente.

$\mathrm{Al}$ respecto de la presencia de esta combinación ortográfica ui nos parece muy oportuno transcribir todos los apartes de Tomás Navarro de su párraf̣o 149, de su obra citada.

El grupo acentuado ui. Las palabras en que aparece este grupo pueden considerarse divididas, por lo que a la pronuncición se refiere, en tres secciones distintas: 
a. Palabras en que la combinación ui, cualquiera que sea su origen, se pronuncia como diptongo, wi: juicio, buitre, cuita, benjú, muy, cuido, cuidas, fuí, fuiste, etc. El arcaísmo ju-icio sólo por excepción suele hoy encontrarse alguna vez en los poetas. La pronunciación antigua de las formas muy, cuita, cuida, cuide, etc., con acento sobre la $u$, ú, a la cual se hizo ya alusión, y 66, $n$., es aún corriente en Asturias y en algunos otros puntos del Norte de España: múi, kúit, kúid. Bello la indicó asimismo como conservada en Chile. La forma corriente y general es evidentemente wi. Un poeta no podría hoy como en otro tiempo, emplear cuida en rima con muda o duda, sino con mida, vida, etc.

Donde ninguno de ninguno cuida pronto se aprende a conocer la vida. (Espronceda)

De mi mano cuida... / Que le dio medida. (E. Marquina)

$b$. Palabras que se pronuncian con diptongo, wi, y con hiato, $u-i$, dándose preferencia al diptongo: ruido, ruin, ruina, arruino, arruina, suizo, circuito, fortuito, gratuito, casuista, etc. La forma corriente en cualquier caso, hasta en posición final, es el diptongo. El hiato, tratándose de estas palabras, responde en general a una pronunciación más cuidada y escogida que la de la lengua ordinaria. En ruido, ruin, ruina, y suizo, el hiato es menos raro que en circuito y fortuito; en gratuito ocurre con relativa frecuencia. En el verso aparece más veces que en la conversación, y en los poetas antiguos más que en los modernos. Hay diferencias individuales; unas personas emplean el diptongo con más regularidad que otras. (...).

c. Palabras que se usan con hiato, $u-i, y$ con diptongo, wi, predominando el hiato: jesuíta, huída, huír, incluír, concluír, concluído, recluido, construí, instruíste, susbstituímos, retribuísteis, etc. Las mismas personas que en una lectura ordinaria y normal dicen rwi-do, swi-@o, etc., en igualdad de circunstancias pronuncian xe-su-i-ta, $u$-i-da, $u$-ìr, in-klu-ír, kon-klu-í-do, etc. ${ }^{4}$

No sabemos si por los tiempos cuando Tomás Navarro escribió su Manual era más frecuente en toda España, como lo es hoy, y no solamente en Asturias y el Norte, como él anota, pronunciar mui, hoy pronunciación arcaica, según el mismo autor. De otra parte no hace referencia don Tomás en parte alguna, sobre lo que al respecto pudiera estar ocurriendo, en general, en América, donde los millones de hablantes son abrumadora mayoría en la comunidad hispánica. 
De todas maneras, y por conclusión, no compartimos que la pronunciación moderna para muy debe ser normativamente [mwi] y que la pronunciación decreciente [mui] hoy sea sólo una pronunciación antigua en algunas partes de España; lo mismo puede sostenerse con respecto a cuy, Ruy, Cocuy, Jujuy, huy, Ibicuy, donde nos ha parecido siempre que la realización de $u y$ es, americanamente, el diptongo decreciente $[u i]$ y no el creciente [wi].

Nos parecio imprescindible para el propósito de este trabajo ilustrar aquí lexicográficamente la existencia y uso de ejemplos que contienen el diptongo [ui] en palabras mencionadas y pronunciadas por diferentes personas de procedencia hispanoamericana; recurrimos a las embajadas de estos países en Bogotá a fin de encontrar personas de esas procedencias, las cuales suministraron el material lingüístico antes mencionado, así:

Colombia. Mūy, Cocūy, Rūy, Tỉbacūy (topónimo), Tilagūy (apellido), hūy, cūy (curí o conejillo de Indias), jūy (interjección), chichagūy (forúnculo), currucūy (buho).

Venezuela. (Informante: Maestra Mayor Carlos Rodríguez), Tưy (valle, río, diosa indígena), Tepūy (volcán), Cocūy (ron, bebida similar al pulque o mezcal mexicanos, extraído del maguey o cabuya).

México. (Informante: Gilberto Limón): hūy, mūy.

Argentina. (Informante: Rolando E. Oviedo): Cocūy = cocuyo, cūy = cobayo, Ibicūy (topónimo), Jujūy (provincia), machicūy (familia lingüística).

Chile. (Informante: Marina Bonatti): hūy, mūy.

Panamá. (Informante: Edison Samaniego): hūy, mūy.

Bolivia. (Informante: René Carvajal): hūy, mūy, Villa Azurdūy.

Nicaragua. (Informante: Chanta Palé): mūy,hūy.

Perú. (Informante: Susana Fajardo): hừy, mūy, cūy.

Honduras. (Informante: Douglas Licona): mūy, hūy.

Paraguay. (Informante: Enrique Ruiz): hūy, mūy.

Ecuador. (Informante: Miriam Jácome): hūy, mūy, cūy, Puerto Chandūy (topónimo).

Ilustrado suficientemente este aparte pasaremos al planteamiento mismo del problema central. 


\section{PLANTEAMIENTO DEL PROBLEMA}

Propósito específico.

Por lo visto en todas las explicaciones anteriores, se delimitan ahí dos situaciones muy específicas: los diptongos hispánicos son 14 según la consideración que le da a tal fenómeno la investigación tradicionalista desde don Tomás Navarro hasta Quilis, pasando por Gili Gaya, Alarcos y Salvador Rodríguez; o los diptongos hispánicos son, en realidad, 15 teniendo en cuenta que la concurrencia ortográfica ui, no solamente se realiza como diptongo creciente [wi], como fui-mos o como hiato (hu-í-mos), sino también como diptongo decreciente [ui] en una serie de palabras, que entre otras cosas, si bien no es inmensa tiene la representación necesaria de términos generales y locales que se conocen y se manejan lingüísticamente en el área de la lengua hispánica.

Ante ello, no nos queda otra pretensión, apenas lógica, que entrar a indagar con evidencias de la fonética experimental, si los informantes - cuidadosamente seleccionados para el suministro del material por examinar - proporcionaron unas muestras acústicas en las cuales, tanto por análisis impresionístico, como por análisis experimental-matemático se puede probar o no la presencia de este tipo de diptongo. Como paso inicial fue necesario preparar un material lingüístico a manera de corpus, el cual debió ser grabado por cada uno de los informantes. Veámoslo.

\section{El material lingüístico.}

Se tomaron los siguientes cinco términos básicos: muy (adverbio), Huy (interjección), cuy (conejillo de Indias), Cocuy (topónimo o agave, pita, maguey o cabuya, del cual se obtiene el cocuy masato venezolano o el mezcal y pulque mexicanos), y Ruy (nombre hispánico). Con ellos se elaboraron estas cinco oraciones breves.

1. Vino muy cansado.

2. iHuy qué horror!

3. Carne de cuy.

4. La sierra del Cocuy.

5. Ruy Díaz, El Cid.

Los informantes.

Para la escogencia de los 20 informantes se tomaron los criterios de alfabetismo; procedencia, edad cronologica y sexo. Para la primera condición se cubrieron los 
siguientes niveles, así: 1 . Con postgrado; 2 . Con título universitario; 3 . Con estudios preuniversitarios o algunos semestres de universidad; 4 . Con bachillerato y 5 . con estudios primarios completos; en cada uno de estos niveles (A-B-C-D-E) se escogieron 4 informantes. Para cumplir con el criterio de edad, se balancearon los 20 informantes en dos posibilidades: de 20 a 45 años y de más de 45 años y entre estas condiciones se eligió hombre y mujer para equilibrar la representacion masculina y femenina. Veamos el siguiente cuadro que nos dará una idea más clara de la conformación equilibrada de la escogencia de los informantes.

\begin{tabular}{|c|c|c|c|c|c|}
\hline Grupos & A & B & C & D & E \\
\hline Edad & Con postgrado & $\begin{array}{c}\text { Con titulo } \\
\text { universitario }\end{array}$ & $\begin{array}{c}\text { Con } \\
\text { universidad }\end{array}$ & $\begin{array}{c}\text { Con } \\
\text { bachillerato y/o } \\
\text { Est.Preuniver }\end{array}$ & $\begin{array}{c}\text { Con primaria o } \\
\text { parte }\end{array}$ \\
\hline Más de 45 años & $\begin{array}{c}\text { Ma.Luisa de } \\
\text { Mentes, Jaime } \\
\text { Bernal }\end{array}$ & $\begin{array}{c}\text { Melba } \\
\text { Aristizabal, } \\
\text { César } \\
\text { Navarrete }\end{array}$ & $\begin{array}{c}\text { Carmenza } \\
\text { Muñoz, Jose } \\
\text { Eduardo } \\
\text { Jimenez }\end{array}$ & $\begin{array}{c}\text { Victoria Pardo, } \\
\text { Guillermo } \\
\text { Escallon }\end{array}$ & $\begin{array}{c}\text { Ma.Cristina } \\
\text { Rueda, Moisés } \\
\text { Morara }\end{array}$ \\
\hline Ernesto Porras & $\begin{array}{c}\text { Alcira } \\
\text { Valencia, } \\
\text { Nestor Valencia }\end{array}$ & $\begin{array}{c}\text { Jenny de } \\
\text { Calderon, } \\
\text { Ismael Delgado }\end{array}$ & $\begin{array}{c}\text { Graciela } \\
\text { Granados, } \\
\text { Carlos } \\
\text { Echavarría }\end{array}$ & $\begin{array}{c}\text { Ligia de } \\
\text { Gamba, } \\
\text { Manuel Tapias }\end{array}$ \\
\hline
\end{tabular}

Muestra acústica: Elaboración y descripción.

Las muestras acústicas, sonogramas en este caso, fueron elaboradas por el espectrografo 6067B de la Kay Elemetrics, en banda ancha y con amplitude display, o curva de intensidad, recurso este que da la mejor posibilidad de examinar el mayor decibelaje en un segmento dado, aspecto que es justamente el que vamos a analizar en las curvas correspondientes del segmento /ui/ en cada texto. Estos amplitude displays fueron realizados en banda estrecha a fin de posibilitar una línea más fina de visualizar y de poder medir en decibeles. Logrados los sonogramas, procedimos a su segmentación y su transcripción correspondiente. Adelante se mostrarán algunos de los sonogramas más representativos de los 45 examinados; lo anterior, obviamente por razones de espacio editorial.

Todo el trabajo de fonética acústica experimental de esta investigación se realizó en los laboratorios de fonética del Instituto Caro y Cuervo (Yerbabuena) de la República de Colombia.

\section{Muestras seleccionadas.}

En forma previa se llevaron al sonógrafo entre dos y tres muestras grabadas de cada uno de los informantes escogidos, con el equilibrio que puede observarse en los siguientes cuadros estadísticos. 


\begin{tabular}{|c|c|c|c|c|c|}
\hline \multicolumn{6}{|c|}{ GRUPO A } \\
\hline Informante & $\begin{array}{c}\text { Vino muy } \\
\text { cansado }\end{array}$ & $\begin{array}{c}\text { Huy que } \\
\text { horror }\end{array}$ & $\begin{array}{c}\text { Carne de } \\
\text { cuy }\end{array}$ & $\begin{array}{l}\text { La sierra } \\
\text { del Cocuy }\end{array}$ & $\begin{array}{c}\text { Ruy Díaz, } \\
\text { El Cid }\end{array}$ \\
\hline \multicolumn{6}{|l|}{ Texto } \\
\hline Ma. Luisa de Montes & $x$ & & $\mathrm{x}$ & & \\
\hline Jaime Bernal & & $\mathbf{x}$ & $\mathbf{x}$ & $\mathbf{x}$ & \\
\hline Jenny Figueroa & $\mathbf{x}$ & & $\mathbf{x}$ & & \\
\hline Ernesto Porras & $\mathbf{x}$ & $\mathbf{x}$ & & & \\
\hline \multicolumn{6}{|c|}{ GRUPO B } \\
\hline Informante & T. 1 & T. 2 & T. 3 & T. 4 & T. 5 \\
\hline \multicolumn{6}{|l|}{ Texto } \\
\hline Melba Aristizabal & & $x$ & $x$ & & \\
\hline César Navarrete & $\mathbf{x}$ & $\mathbf{x}$ & & & \\
\hline Alcira Valencia & & $\mathbf{x}$ & & $\mathrm{x}$ & \\
\hline Nestor Valencia & $x$ & $x$ & & & $\mathrm{x}$ \\
\hline \multicolumn{6}{|c|}{ GRUPO C } \\
\hline Informante & $\mathrm{T} .1$ & T. 2 & T. 3 & T. 4 & T. 5 \\
\hline \multicolumn{6}{|l|}{ Texto } \\
\hline Carmenza Muñoz & $x$ & & $\mathbf{x}$ & & $x$ \\
\hline José Eduardo Jiménez. & $\mathbf{x}$ & & & & $\mathbf{x}$ \\
\hline Jenny de Calderon & & & $\mathrm{x}$ & $\mathrm{x}$ & \\
\hline Ismael Delgado & $\mathbf{x}$ & & $x$ & & \\
\hline \multicolumn{6}{|c|}{ GRUPO D } \\
\hline Informante & T. 1 & T. 2 & T. 3 & T. 4 & T. 5 \\
\hline \multicolumn{6}{|l|}{ Texto } \\
\hline Victoria Pardo & $\mathbf{x}$ & $\mathrm{x}$ & & $\mathrm{x}$ & \\
\hline Guillermo Escallón & . & $\mathbf{x}$ & & $\mathbf{x}$ & \\
\hline Ligia de Gamboa & . & $\mathbf{x}$ & & & $\mathbf{x}$ \\
\hline Carlos Echavarría & & & $\mathbf{x}$ & $\mathrm{x}$ & \\
\hline \multicolumn{6}{|c|}{ GRUPO E } \\
\hline Informante & T. 1 & T. 2 & T. 3 & T. 4 & T. 5 \\
\hline \multicolumn{6}{|l|}{ Texto } \\
\hline Ma.Cristina Rueda & $\mathbf{x}$ & 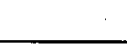 & $\mathrm{x}$ & & \\
\hline Moises Mora & & & & $x$ & $x$ \\
\hline Graciela Granados & $x$ & & & $\mathbf{x}$ & $\mathbf{x}$ \\
\hline \multirow[t]{2}{*}{ Manuel Tapias } & & $x$ & & & $\mathbf{x}$ \\
\hline & 11 & 10 & 9 & 8 & 7 \\
\hline
\end{tabular}


A cada uno de los grupos le sonografiamos 9 grabaciones (45 en total).

Al texto Vino muy cansado se le hicieron 11 sonogramas; al texto 2, 10 sonogramas; al texto 3, 9 sonogramas; al texto 4, 8 sonogramas y al texto 5, 7 sonogramas $(=45)$. Pensamos que a los textos que contenían muy - huy - cuy, por ser estos vocablos, palabras, en su orden, con mucho más ámbito geográfico y de mayor uso, debían sonografiarse más veces, y menos veces los textos con Cocuy y $R u y$ por razones obvias. Pero de todas maneras se brindó aquí la oportunidad de analizar igual número de sonogramas de cada grupo de informantes, así como de los 4 informantes con tres sonogramas cada uno, dos correspondieron a caballeros y dos a damas. Nos parece, pues, que, en general, hemos atendido a un especial equilibrio de condiciones.

\section{MEDICIÓN Y RESULTADOS ESTADÍSTICOS EXPERIMENTALES}

Se utilizaron las muestras seleccionadas (45 en total) como material de medición.

Apartándonos del método impresionístico, (o sea, observación del mayor ennegrecimiento del segmento acústico $u i$ ) procedimos por razones mucho más científicas y experimentales a tomar la curva superior del segmento [ui] en el amplitude display correspondiente, en cada uno de los 45 sonogramas. En ellas se observó a cuál de los dos fonos vocálicos $[u i]$ correspondía la mayor elevación de la curva, lo cual sería en finales, índice inequívoco de su mayor intensidad. De los 5 cuadros originales (uno de cada grupo de informantes) mostramos a continuación c/u de ellos (grupos $\mathrm{ABCDE}$ ). Puede verse indiscutiblemente en estos gráficos la profusión de muestras, (curvas de amplitude display) en donde la mayor elevación (es decir, mayor intensidad, o sea, vocal nuclear yno semivocal) corresponde a la vocal $u$ y no a la $i$. Todo para probar experimentalmente la ejecución de un diptongo [ul] decreciente y no [wi] creciente. Igualmente de los 45 sonogramas elaborados decidimos seleccionar 5 para este artículo (uno de cada grupo), para mostrar con más recursos la comprobación del mismo fenómeno pero a nivel espectrográfico.

Obsérvense, pues, tanto los cinco cuadros de amplitude display y los cinco sonogramas y léanse con detenimiento la breve descripción en la base de cada uno de estos.

Cuadros analíticos de los segmentos intensivos.

Examinados los cinco cuadros de amplitude display de los 45 sonogramas a nivel intensivo, resultaron estas evidencias:

- Símbolo: + Resultado positivo, es decir, diptongo [ui]

- Símbolo: - Resultado negativo, es decir, diptongo [wi] 


\section{TEXTOS}

\begin{tabular}{|c|c|c|c|c|c|c|c|}
\hline Grupos & Informante & $\begin{array}{l}\text { Vino muy } \\
\text { cansado }\end{array}$ & $\begin{array}{l}\text { Huy qué } \\
\text { horror }\end{array}$ & $\begin{array}{l}\text { Carne de } \\
\text { cuy }\end{array}$ & $\begin{array}{l}\text { La sierra } \\
\text { del Cocuy }\end{array}$ & $\begin{array}{c}\text { Ruy Díaz } \\
\text { El Cid }\end{array}$ & $\begin{array}{c}\text { Totales } \\
\text { Transitorios }\end{array}$ \\
\hline \multirow{5}{*}{$\mathbf{A}$} & 1 & + & & + & & & \\
\hline & 2 & & + & + & + & & \\
\hline & 3 & + & & - & & & \\
\hline & 4 & - & + & & & & \\
\hline & Subtotal & $\begin{array}{c}2+/ 1- \\
(66 \%+)\end{array}$ & $\begin{array}{c}2+/ 0- \\
(100 \%+)\end{array}$ & $\begin{array}{c}2+/ 1- \\
(66 \%+)\end{array}$ & $\begin{array}{c}1+/ 0- \\
(100 \%+)\end{array}$ & & $\begin{array}{c}7+/ 2- \\
(79 \%+)\end{array}$ \\
\hline \multirow{5}{*}{ B } & 1 & & + & + & & & \\
\hline & 2 & + & + & & & & \\
\hline & 3 & & + & & + & & \\
\hline & 4 & + & - & & & + & \\
\hline & Subtotal & $\begin{array}{c}2+/ 0- \\
(100 \%+)\end{array}$ & $\begin{array}{c}3+/ 1- \\
(75 \%+)\end{array}$ & $\begin{array}{c}1+/ 0 \\
(100 \%+)\end{array}$ & $\begin{array}{c}1+/ 0 \\
(100 \%+)\end{array}$ & $\begin{array}{c}1+/ 0- \\
(100 \%+)\end{array}$ & $\begin{array}{c}8+/ 1- \\
(95 \%+)\end{array}$ \\
\hline \multirow{5}{*}{ C } & 1 & + & & + & & + & \\
\hline & 2 & + & & & & & \\
\hline & 3 & & & + & + & + & \\
\hline & 4 & + & & + & & & \\
\hline & Subtotal & $\begin{array}{c}3+/ 0 \\
(100 \%+)\end{array}$ & & $\begin{array}{c}3+/ 0- \\
(100 \%+)\end{array}$ & $\begin{array}{c}1+/ 0- \\
(100 \%+)\end{array}$ & $\begin{array}{c}2+/ 0- \\
(100 \%+)\end{array}$ & $\begin{array}{c}9+/ 0 \\
(100 \%+)\end{array}$ \\
\hline \multirow{5}{*}{ D } & 1 & + & - & & + & & \\
\hline & 2 & & + & & + & & \\
\hline & 3 & & + & & & + & \\
\hline & 4 & & & + & + & & \\
\hline & Subtotal & $\begin{array}{c}1+/ 0- \\
(100 \%+)\end{array}$ & $\begin{array}{c}2+/ 1- \\
(66 \%+)\end{array}$ & $\begin{array}{c}1+/ 0- \\
(100 \%+)\end{array}$ & $\begin{array}{c}3+/ 0- \\
(100 \%+)\end{array}$ & $\begin{array}{c}1+/ 0- \\
(100 \%+)\end{array}$ & $\begin{array}{c}8+/ 1- \\
(93 \%+)\end{array}$ \\
\hline \multirow{5}{*}{$\mathrm{E}$} & 1 & + & & + & & & \\
\hline & 2 & & & & + & + & \\
\hline & 3 & - & & & + & + & \\
\hline & 4 & & + & & & + & \\
\hline & Subtotal & $\begin{array}{c}1+/ 1- \\
(50 \%+)\end{array}$ & $\begin{array}{c}1+/ 0- \\
(100 \%+)\end{array}$ & $\begin{array}{c}1+/ 0- \\
(100 \%+)\end{array}$ & $\begin{array}{c}2+/ 0- \\
(100 \%+)\end{array}$ & $\begin{array}{c}3+/ 0- \\
(100 \%+)\end{array}$ & $\begin{array}{c}8+/ 1- \\
(90 \%+)\end{array}$ \\
\hline Total defin. & 25 inform. & $\begin{array}{c}9+/ 2- \\
(81 \%+)\end{array}$ & $\begin{array}{c}8+12- \\
(80 \%+)\end{array}$ & $\begin{array}{c}8+/ 1- \\
(88 \%+)\end{array}$ & $\begin{array}{c}8+/ 0- \\
(100 \%+)\end{array}$ & $\begin{array}{c}7+/ 0- \\
(100 \%+)\end{array}$ & $\begin{array}{c}40+/ 5- \\
(88 \%+)\end{array}$ \\
\hline
\end{tabular}


Para la elaboración del anterior cuadro estadístico tomamos los cinco grupos de informantes y sus respectivos sonogramas seleccionados (45 muestras). En éstos analizamos los segmentos intensivos en el amplitude display, correspondientes logicamente a los presuntos diptongos [ui]. Si la mayor elevación o cúspide intensiva correspondía a [ $u$ ], marcamos,+ y -, si la mayor cúspide correspondía a [i].

De las once muestras de muy examinadas, 9 tuvieron más intensidad en $[u]=$ $81 \%+$. En las 10 muestras de huy, 8 fueron positivas $=80 \%+$. De 9 muestras de cuy, en 8 se hallo a $[u]$ con mayor intensidad o cúspide más alta $=88 \%+$; en las 8 muestras de Cocuy todas presentaron el segmento $[u]$ con cúspide más alta; finalmente, las 7 muestras de Ruy presentaron diptongo decreciente, es decir, con mayor decibelaje para $[u]$.

Globalizando, de las 45 muestras examinadas, 40 mostraron mayor elevación del segmento amplitude display en el sector correspondiente a $[u]$ y 5 , mayor cúspide para [i]. El porcentaje sería, pues, de un $88 \%$ a favor de la presencia del diptongo [ui] y un $12 \%$ a favor del diptongo [wi], en 45 muestras con 20 informantes.

GRUPO "A“

\begin{tabular}{|l|l|l|l|l|l|}
\hline Vino muy cansado & Huy que horror & Carne de cuy & La sierra del Cocury & Ruy Diaz El Cid \\
\hline Ma. Luisa de Montes & & & & & \\
\hline
\end{tabular}




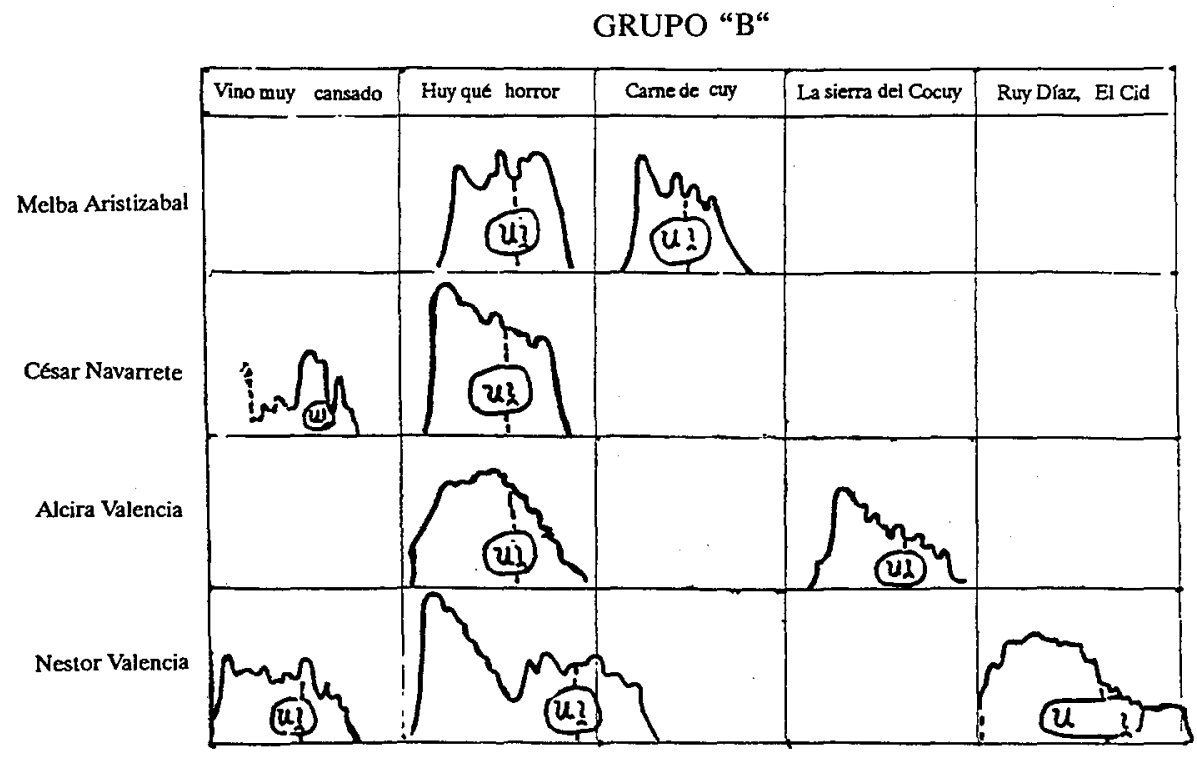

\begin{tabular}{|l|l|l|l|l|l|}
\hline Vino muy cansado & Huy que horror & Came de cuy & La sierra del Cocury & Ruy Dlaz, El Cid \\
\hline Jenny de Calderon & & & & \\
\hline
\end{tabular}


GRUPO “D”

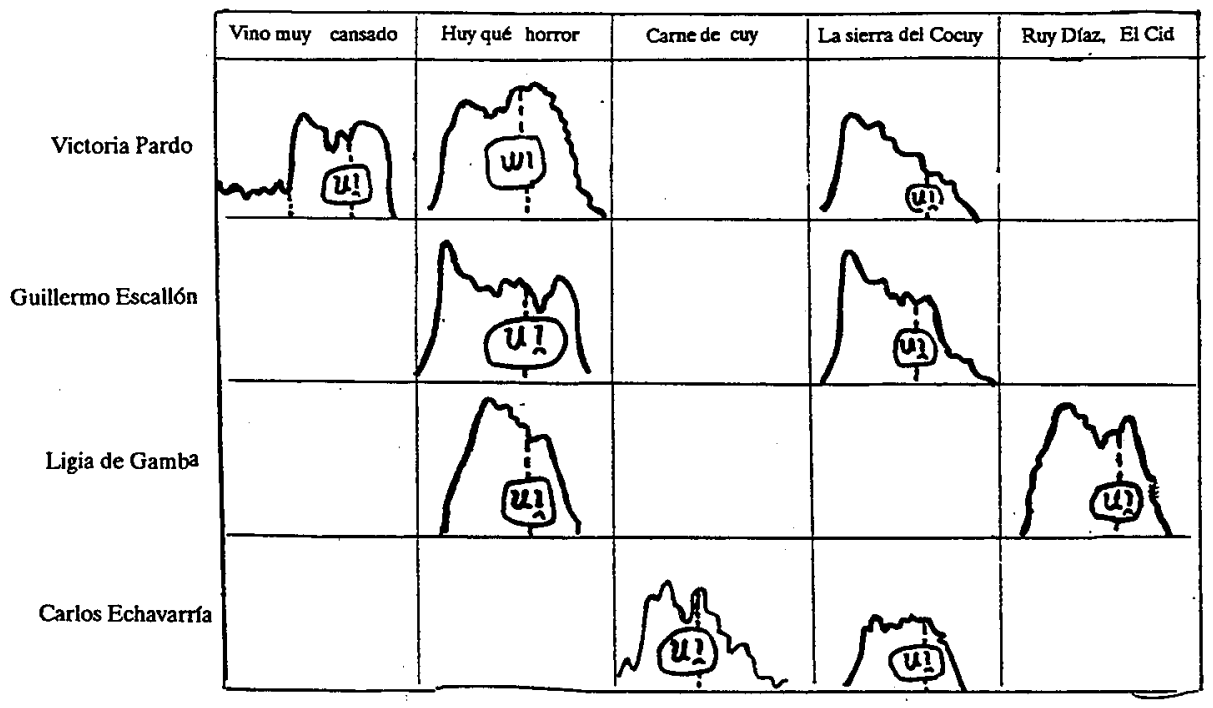

GRUPO "E"

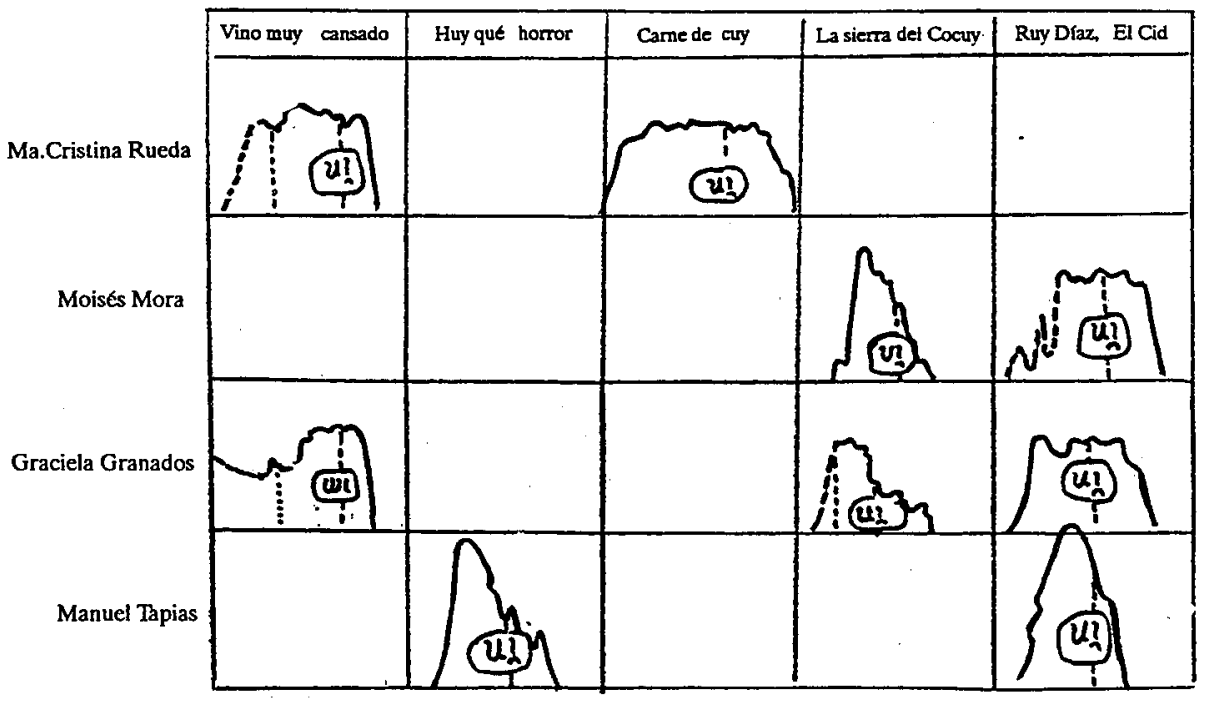

82 
Por la época cuando desarrollábamos esta investigación en 1984, pasaba el Dr. Germán de Granda (español) una temporada académica en El Seminario Andrés Bello del Instituro Caro y Cuervo. Nos parecio muy oportuno aprovechar al informante mencionado para que nos proporcionara la grabación del mismo material utilizado con los otros 20 informantes del presente trabajo.

Lo anterior como una muestra no hispanoamericana de lo que suponíamos podía ser también en España un nuevo diptongo decreciente, es decir [ $\left.u i{ }_{i}\right]$.

De otra parte, creímos conveniente verificar con algunas personas españolas residentes en Bogotá, cuál era la ejecución fónica de la concurrencia [uị], con los siguientes resultados:

PACO SANCHEZ: mūy - hūy - Rūy

MATILDE TERRESSE DE HOFFMAN: mūy - hūy - Rūy

MARIBEL VELASQUEZ MIRANDA DE HENAO: mūy - hūy - Rūy

Impresionísticamenrte les escuchamos a estos españoles siempre diptongos decrecientes.

A continuación se incluyen los 5 segmentos intensivos (amplitude displays) extraídos de los sonogramas elaborados con el material lingüístico grabado por el Dr. Germán de Granda. En todas ellas también puede verse evidentemente un mayor decibelaje, una mayor elevación de la curva en el sector que corresponde a u.

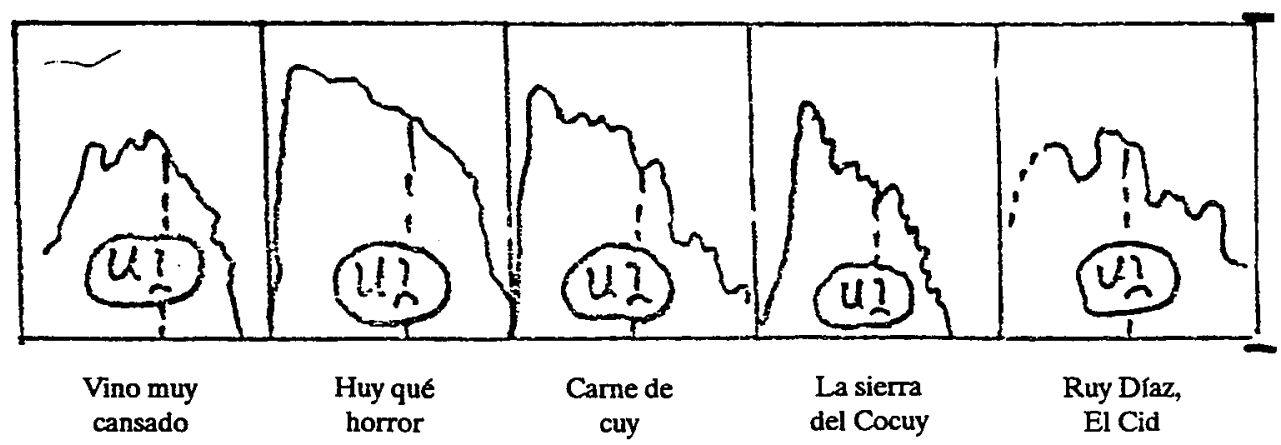




\section{CONCLUSIONES}

El diptongo [ui] existe plena y confirmadamente en el mundo hispánico. Si es bien cierto que en algunos sectores geográficos de España existe la modalidad [wi] para la concurrencia / $u$ i/ de palabras como muy, huy, Ruy, etc., iberoamericanos y peninsulares prefieren abrumadoramente [ui] y esta realización alofónica es del dominio y uso del mundo hispanoparlante.

No solo la impresión acústica de muchos interrogados y de los encuestados, sino la existencia de palabras de vieja raíz peninsular así como otros regionalismos americanos y no pocos colombianismos (tal vez de origen chibcha, es decir, misquismos) que desde un comienzo mostraron casi que sin lugar a duda alguna la mayor audibilidad en $u$ que en $i$, nos colocaron en una situación privilegiada para entrar a probar acústicamente y con métodos matemáticos experimentales la misma evidencia.

Comprobar en los amplitude displays a cuál de los fonos correspondía el mayor decibelaje fue tarea sencilla, pues inclusive, sin ayuda del estimativo milimétrico, es decir a simple ojo, resulta de gran rendimiento y confiabilidad, determinar con simple método impresionístico, cuál de los dos fonos es más intenso, observando simplemente cuáles son las cúspides más altas.

En conclusión final, la lengua hispánica tiene ya un décimo quinto diptongo, decreciente en este caso, constituído por dos vocales cerradas, $U+I$, de las cuales la $U$ es el fonema central o vocal plena y la $I$ es el fonema marginal de características semivocálicas. 


\section{Bibliografía}

Navarro, Tomás, Lecciones de Pronunciación, Madrid, Consejo Superior de Investigaciones Científicas, Gráficas Monteverde, 1968.

Quilis, Antonio, Curso de Fonética y Fonología Españolas, Madrid, Consejo Superior de Investigaciones Científicas, Instituto Miguel de Cervantes, Collectánea Phonetica II, 1971.

Alarcos L1., Emilio, Fonología Española, Madrid, Ed. Gredos, S.A. B.R.H., 1971.

Gili Gaya, Samuel,Elementos de Fonética General, Madrid, Ed. Gredos, B.R.H., 1961.

Lázaro Carreter, Fernando, Diccionario de Términos Filológicos, Madrid, Ed. Gredos, 1962.

Lewandowski, Theodor, Diccionario de Lingüística, Madrid, Ed. Cátedra S.A. $1^{\text {a }}$ traducción de María Luz García y otros.

Dubois, Jean, Diccionario de Lingüística, Madrid, Ed. Alianza, 1979. Traducción de Inés Ortega y otros.

Abraham, Werner, Diccionario de Terminología Linguística Actual, Madrid, Ed. Gredos, 1981. Versión española, Francisco Meno Blanco. 


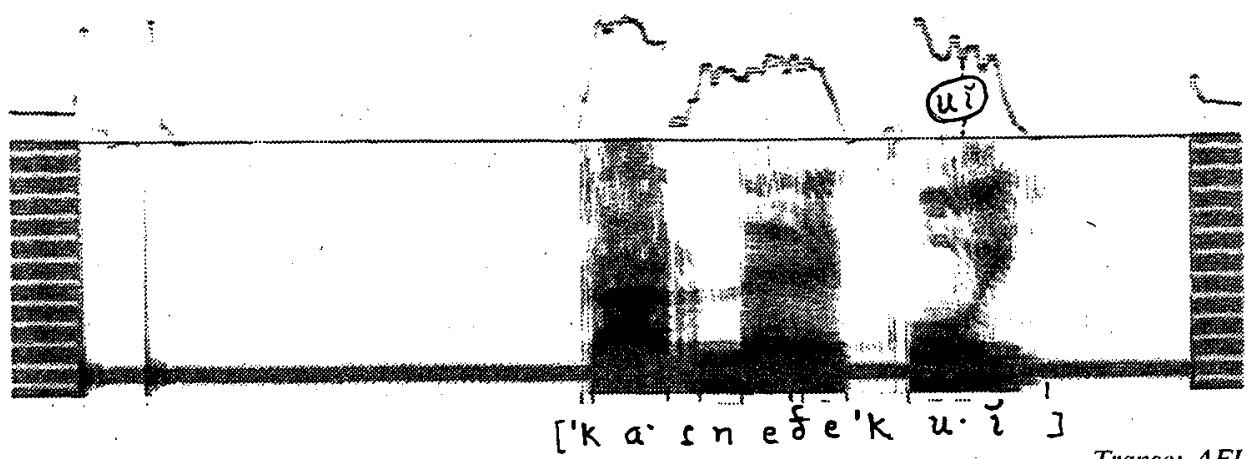

CARNE DE CUY (18). - El segmento [u], muy ennegrecido, tiene una curva muy por encima en d8 frente a la curva de [i]. No hay, pues, razón para afirmar aquí la realizacion de un dipıongo [wĩ], donde la [i]tendria, como seria lógico, mayor decibelaje y mayor ennegrecimiento.

TYPE B/65 SONAGRAM ${ }^{\text {(A) }}$ KAY ELEMETRICS CO. PINE BROOK. N. J.

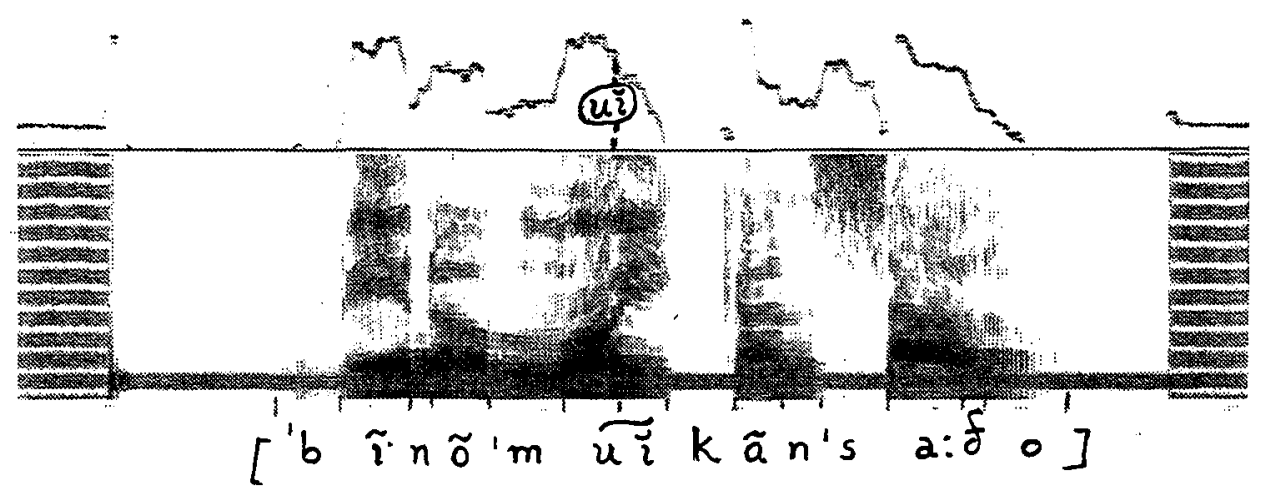

Transc: $A F I$

VINO MUY CANSADO. (4C). - Obsérvese el mayor ennegrecimienıo del segmento acústico [u] en la banda ancha frente a lo mismo del segmento [i]. Igualmente, arriba, se nota con evidente claridad la diferencia en $\mathrm{d} 8$ que hay a favor del trazo curvilineo para $[\mathrm{u}]$ en demostración de su naturaleza núcleo-silábica $y$ no marginal. 


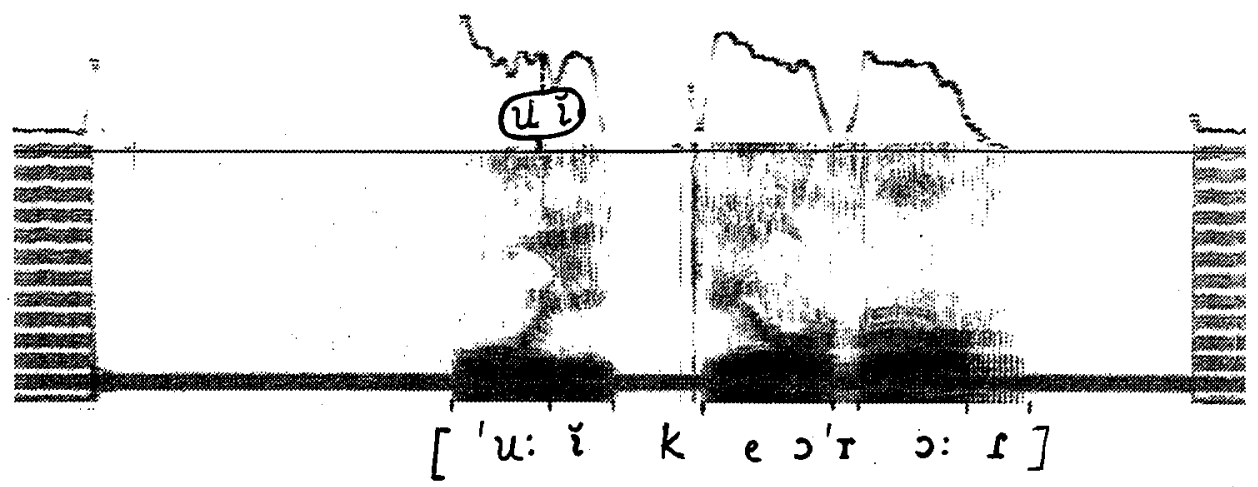

Transc: $A F I$

HUY QUE HORROR (2D). - Obsérvese la mayor intensidad impresionística del segmento [uĩ] así como la mayor duracion del fono núcleo silábico [u]. En el amplitude display bien puede verse la mayor elevación para [u].

TYPE B/65 SONAGRAM ${ }^{\circledR}$ KAY ELEMETRICS CO. PINE BROOK. N. J.

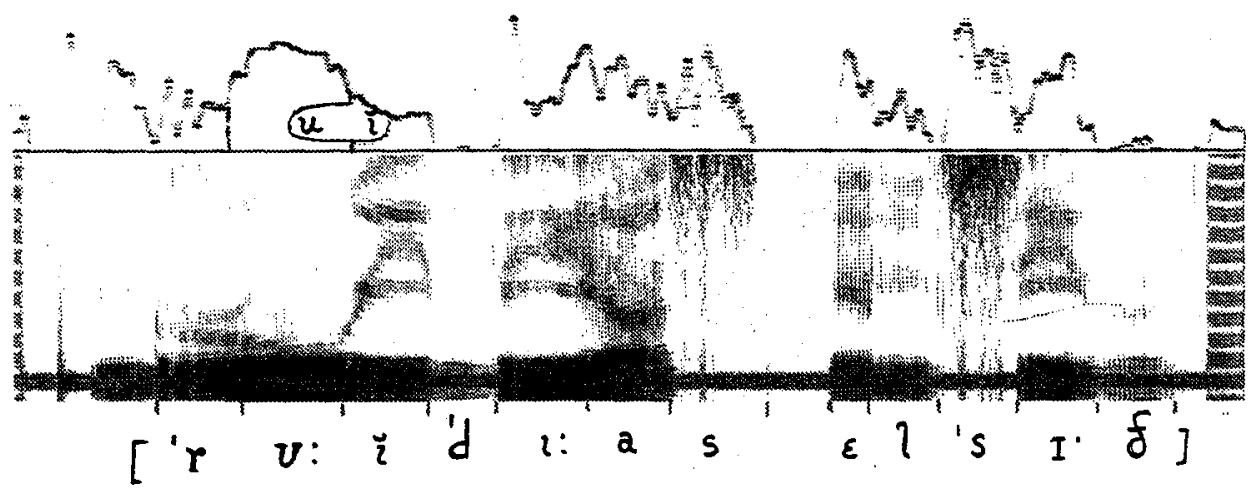

Transc: $A F I$

RUY DIAZ, EL CID (4E). - Tanto duración como elevación de la curva, son mayores para el segmento [u] y menores para [i], constituyendo asi una prueba experimental fehaciente de la existencia de un dipiongo [uY]. 


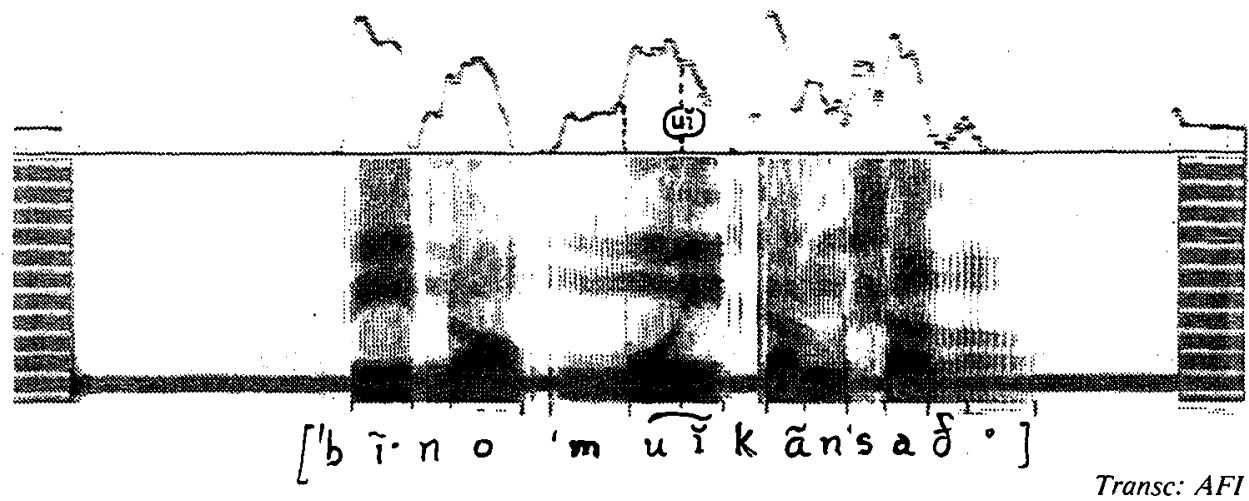

VINO MUY CANSADO (Germán de Granda). No sólo por el mayor ennegrecimiento del segmento [u] (examen comprobatorio de la mayor intensidad con métodos impresionísticos), sino por la presencia de cúspides más altas en el amplitude display correspondiente, se prueba tambien aquí la existencia del diptongo decreciente [uī] en el vocablo MUY.

TYPE B/65 SONAGRAM KAY ELEMETRICS CO. PINE BROOK. N. J.

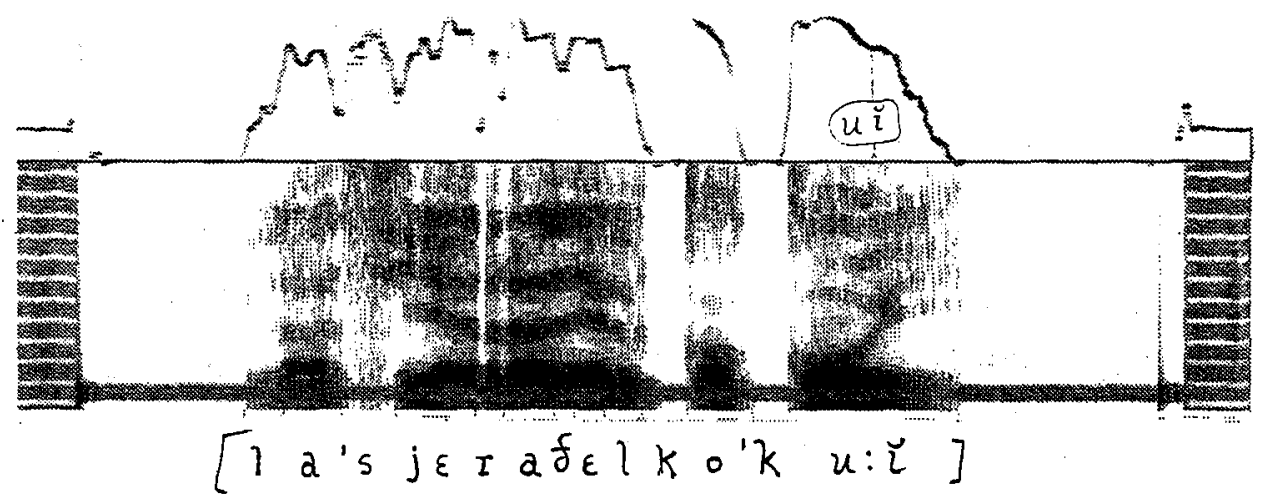

Transc: $A F I$

LA SIERRA DEL COCUY (2A). - ExIraordinaria eviclencia de la mayor carga intensiva del fono [u] en 'cuy'. Nótense, tanto la mayor elevación del amplitude display correspondiente, asi como la mayor concentración especiral de energía en el segmento de banda ancha. 